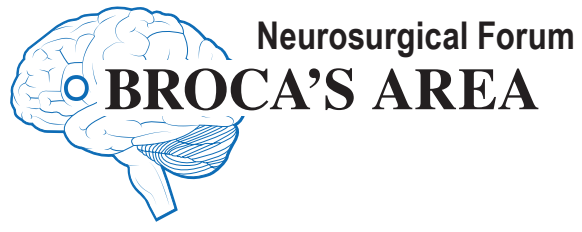

\section{The art of microneurosurgery and passion for technical excellence}

\author{
Aaron A. Cohen-Gadol, MD, MSc, MBA
}

Goodman Campbell Brain and Spine, Department of Neurological Surgery, Indiana University School of Medicine; and Neurosurgical Atlas, Indianapolis, Indiana

$\mathrm{T}$ Dhe technical art of neurosurgery has evolved significantly from the time the discipline was founded. Since then, we have striven to achieve technical excellence. Despite this intense drive, the definition of a technically gifted microneurosurgeon remains elusive. Is it truly an "I know it when I see it" concept? If the most extraordinary desire of our career is undefinable, how can we implement technical metrics to demonstrate our progress toward that goal?

Passionately pursuing self-reflection and absorbing excellence from colleagues are critical for each surgeon's growth and maturation. Metamorphosis from an average to a great surgeon is possible with a durable and fierce devotion to learning; we can always get better no matter how good we are. There exist undefined visuospatial, manual dexterity, and temperamental skills that cannot be effectively absorbed during the residency years unless one has the talent and an insatiable passion to improve during every phase of one's career and with every operation.

The ingredients of technical prowess are intriguing. This fascination has led some surgeons to eagerly watch their national and international colleagues operate, critically review their own operative videos, and attempt alternative and potentially innovative methods to advance their operative finesse and efficiency. The following is a personal reflection of my ongoing journey to understand the mastery of microsurgical techniques.

\section{Maturation of the Microsurgeon During Training and Beyond}

The execution of operative maneuvers is the result of a decision-making process that involves a continuum, ranging from intuitive (subconscious) to analytical (conscious). ${ }^{1}$
This phenomenon is often appreciated among professional athletes; their level of experience is inversely related to their use of analytical and conscious movements. As a resident's level of experience advances, he or she becomes able to switch between analytical and intuitive processes more effectively.

The learning process is markedly accelerated during the time of residency, providing a reasonable medium for studying intraoperative decision-making. The process of a surgeon's technical maturation during residency is likely to involve 3 stages. 1) Before the third year of residency, the operative procedure is interpreted as a series of actions (sequential processing). 2) The senior and chief residents assess the operative situation constantly and form a mental picture; their focus is on pattern recognition (consequential processing). 3) The junior attending surgeons are more likely to feel comfortable with a subconscious or automatic mode of surgical decision-making and have a deeper understanding of the immediate consequences of their intraoperative maneuvers (situational awareness).

The conscious analytical process consumes a significant portion of a resident's mental capacity. This process can overwhelm the attention span (processing power) of the learner and create blind spots; ${ }^{2}$ therefore, the learner might realize the consequences of his or her actions too late in the operative process, which can lead to irreversible results. This sequence of events is likely to be the most common cause of difficult-to-correct surgical errors committed by senior residents.

The expert surgeon deliberately looks for inconsistencies and remains flexible to adjusting the operative plan when necessary. However, the novice surgeon will frequently look for consistencies at the risk of overlooking early, unexpected clues that demand a rearrangement in surgical strategy. Forcing the pathoanatomy to conform to a preplanned operative strategy rather than dynamically revising that strategy in real time to protect neurovascular structures can increase surgical risk significantly and is the most likely but unrecognized cause of intraoperative errors among neurosurgeons.

Operative efficiency decreases the risk of fatigue and resultant stress on the surgeon. The effect of operator fa- 
tigue and burnout on the results of surgery are undoubtedly underestimated. Acute stress can cause tunnel vision and reliance on heuristics due to a decrease in attention span and increase in reaction times. The operator is usually unaware of these effects of fatigue.

\section{Sustainable Pursuit of Technical Excellence}

Master surgeons operate primarily in the subconscious operative mode. Therefore, they enjoy a significant degree of operative efficiency and an expanded attention span, which they can use to focus effectively on operative variations (mismatch between what is expected and what is observed) at each operative moment (variation-detection mode) while attempting resourceful and inventive operative maneuvers. They are comfortable with handling unexpected findings and difficulties expeditiously, so the intrusion of inefficient and risk-averse maneuvers will not lead to extended operative times. I argue that the evolution of technical expertise depends most on proficient variance detection and uncertainty management.

The surgeon's level of self-awareness of his or her strengths is a crucial aspect of recognizing variations in the operative field in a timely fashion to adapt the operative strategy accordingly. Adherence to the original operative strategy to the point of religious conviction can create inefficiencies, operator discomfort, and ultimately undesirable outcomes. The dynamic cycle of situational awareness based on intraoperative findings and adaptive maneuvers evolves the fluidity of surgical flow.

Technical fluency and efficiency are the hallmarks of technical mastery. All the master surgeon's nondeliberate movements are minimized, and his or her operative times are kept to a minimum, even during a particularly difficult surgery (e.g., for complex arteriovenous malformations or skull base tumors). No movement is performed impatiently under time pressure. The time saved is based on the execution of gentle, deliberate, decisive, aesthetically pleasing, and choreographically planned maneuvers and the avoidance of hesitant and wasteful exploratory movements. This operative efficacy can lead to an improved outcome in every case.

Sincere devotion to technical learning plays a pivotal role in enhancing a surgeon's efficiency. Fluent, active, and smooth transitional movements have replaced those that are passive, lethargic, and exploratory. The experience gained from a critical review of one's own surgical videos can eliminate time taken later wondering what to do next during a complex operation.

The master surgeon abandons time-consuming unnecessary approaches and maneuvers, avoids a dogmatic philosophy, and focuses on handling the pathology at hand with minimal disruption of the patient's normal/healthy anatomy. He or she can do more through smaller and more restrictive operative corridors. Flexible operative angles, rather than generous operative space, determine the limits of required exposure.

\section{Technical Innovation That Prioritizes the Patient's Interest}

Technical innovation and, more specifically, the innovative use of operative corridors to deep-seated lesions have been of great interest in academic neurosurgery.
However, this interest must be balanced carefully against the interest of the patient and the true value of the innovation (i.e., its adoption by other surgeons and the steepness of its learning curve).

The capacity of a surgeon to attempt a previously untested operative approach depends on at least 2 factors - that surgeon's work environment, including the support of his or her colleagues (contextual), and an honest assessment of the utility of the new approach (value). The following are 2 brief examples.

Reaching the medial wall of the atrium of the lateral ventricle while avoiding the transparietal approach and the associated risk for visual field defects had been a goal of mine for some time. Work in the dissection laboratory revealed the potential of a transfalcine approach to this region that would result in minimal morbidity; however, this operative route also would have placed the unaffected hemisphere at risk.

At the time, reports on the use of a transfalcine approach had been rare, so the experience with the use of this route for the posterior hemisphere was lacking. Consultations with other surgeons proved this route to be favorable, and as part of a team project we attempted this approach-successfully-in the first patient (Fig. 1). The input of colleagues who had attempted to use similar operative corridors was instrumental in finalizing our plan to proceed. The use of a lumbar drain to minimize retraction on the unaffected hemisphere and localization of the vein of Trolard were some of the basic considerations given during the preoperative planning phase.

We continuously and critically assessed our outcomes after subsequent similar cases, and we applied the same philosophy when we attempted to use another unfamiliar approach. This approach was the paramedian supracerebellar transtentorial amygdalohippocampectomy (in place of an anteromedial temporal lobectomy or selective transsylvian amygdalohippocampectomy), performed in an attempt to preserve the maximal amount of normal dominant temporal lobe (Fig. 2).

In both of these examples, the technical difficulty of the procedure was increased significantly at the expense of a potential benefit to the patient. Because few other surgeons have reported positive experiences with these techniques, the widespread use and value of these complex routes has not yet been demonstrated. True technical innovation is rare and demands a nondogmatic, opportunistic, proactive mentality with a healthy dose of prudence.

The expert or senior surgeon has to maintain his or her passion for technical innovation despite any perceived national distinction. It is often the path of least resistance to become complacent and conform to the expected standards of excellence in the face of increasing administrative responsibilities. Sincere enthusiasm for pursuing excellence is an integral part of every role model for the next generation of neurosurgeons.

\section{Personal Obsession With a Reflective Practice and Nondogmatic Philosophy}

Three factors are critical in the evolution of a surgeon's skills.

1) Objective self-reflection, respect for constructive 

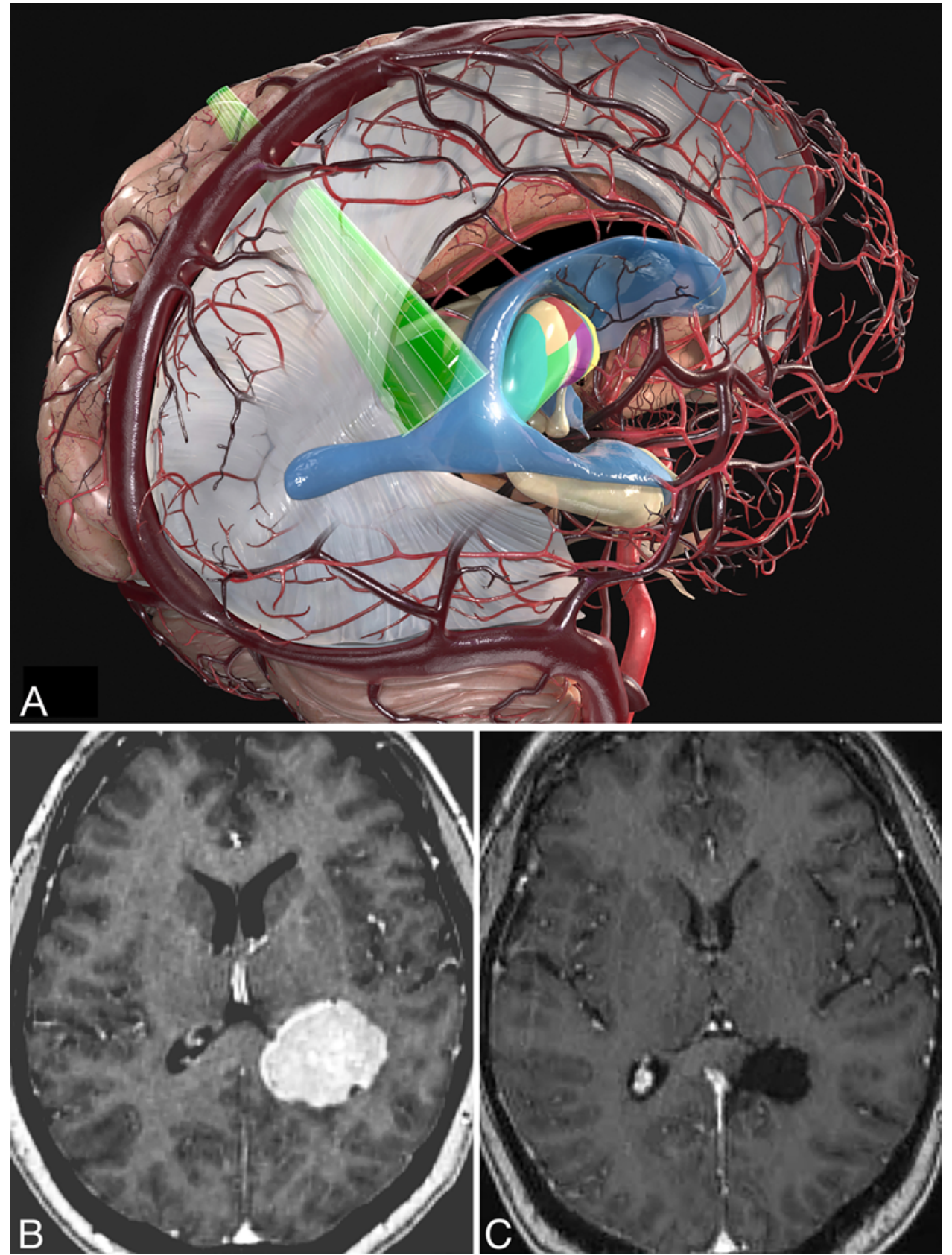

FIG. 1. A: Illustration of the posterior hemispheric transfalcine transprecuneus approach (used with permission from Neurosurgical Atlas by Aaron Cohen-Gadol, MD). Also shown are MR images of an atrial meningioma before (B) and after (C) resection performed via this route, which protected the visual pathways that can be potentially at risk with the transparietal transcortical approach.

feedback from colleagues, and a devotion to learn from one's own as well as colleagues' mistakes and experiences are paramount.

2) We must have a passionate sense of confidence that comes from the knowledge that we, as surgeons, can refine our techniques only by tackling more difficult cases that push the boundaries of our comfort zone. We must be intimately familiar with how to get out of "trouble" to protect the most important result of our work, the welfare of the patient.
3) The key factor in the growth of a microsurgeon's skills is having a visionary spirit to attempt new techniques for difficult-to-reach lesions. All dogma must be abandoned. The rationale for thinking "this is how we always do it" or "this is how I did it in residency" must be questioned.

Rigid loyalty to a particular operative technique can compromise patient care. The surgeon's philosophy should remain flexible, and he or she must consider contingency plans when unforeseen challenges arise. Instead of conforming an operative strategy to the expertise of the sur- 

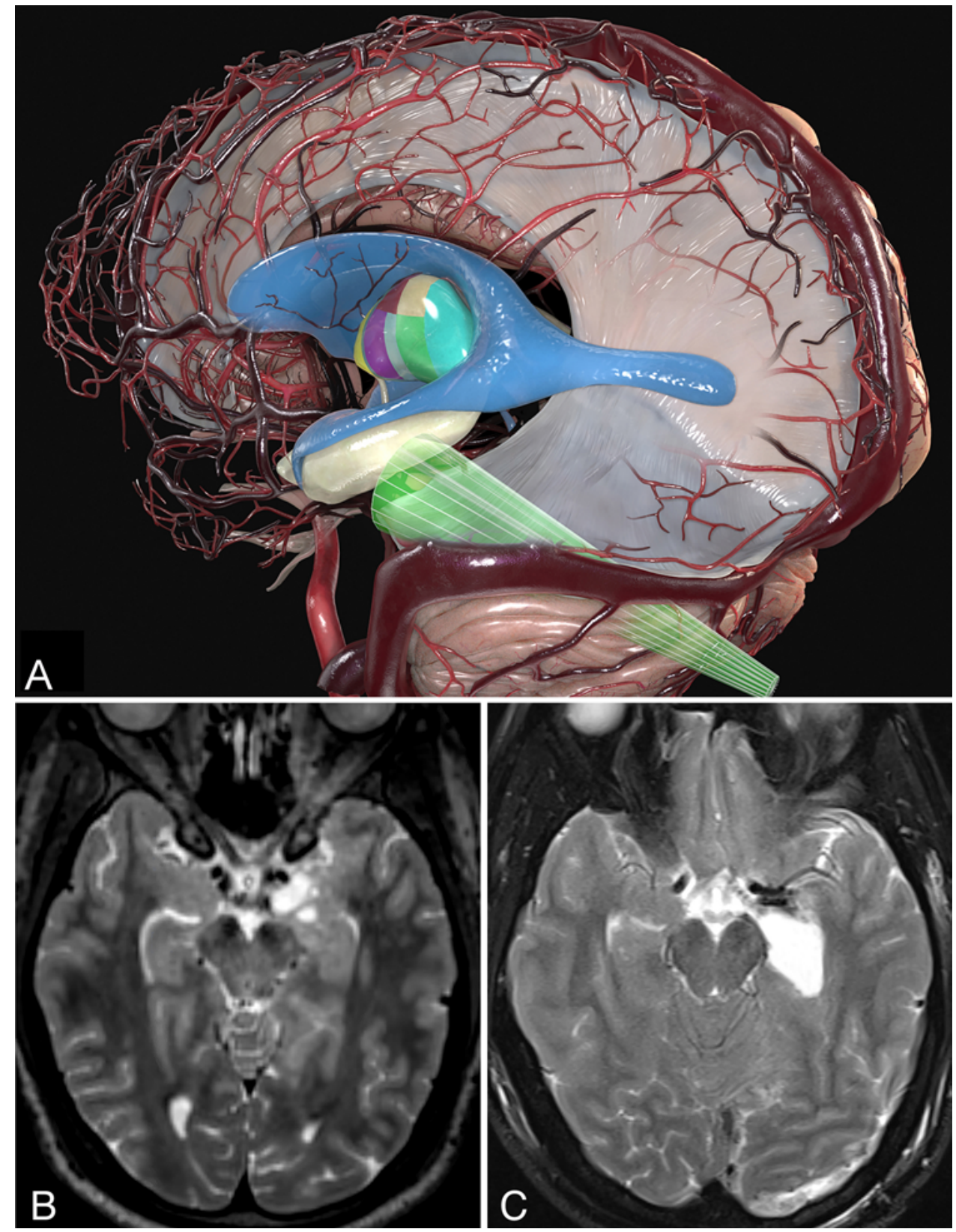

FIG. 2. A: Operative corridor for the paramedian supracerebellar transtentorial amygdalohippocampectomy (used with permission from Neurosurgical Atlas by Aaron Cohen-Gadol, MD). Also shown are MR images of an epileptogenic left medial temporal dysembryoplastic neuroepithelial tumor before $(\mathbf{B})$ and after $(\mathbf{C})$ gross-total resection performed using this operative corridor, which preserved the dominant lateral temporal lobe structures.

geon, the surgeon must revise his or her expertise to match the strategy required for treating the lesion.

Surgical intelligence is the ability to monitor one's own operative maneuvers, discriminate among different operative strategies for handling a lesion efficiently, and use momentous intraoperative findings to guide the overall plan.

Surgeons do not shine when a routine case goes well. However, a master surgeon is recognized when he or she achieves the best outcome under the worst intraoperative circumstances. In other words, the master captain can navigate the ship to the shore under the most turbulent conditions. Surgical intelligence, patience, and an appropriate temperament define the best of us.

Microsurgery is a passion; it is not a job or a hobby but a journey of agony and ecstasy. It can disappoint you, but the successes will carry you along. Most important is that as neurosurgeons, we do "the best that anyone can" rather than "the best that we can" for every patient. Our vocation is a privilege and a badge of honor. 


\section{References}

1. Crebbin W, Beasley SW, Watters DAK: Clinical decision making: how surgeons do it. ANZ J Surg 83:422-428, 2013

2. Francis DMA: Surgical decision making. ANZ J Surg 79:886-891, 2009

\section{Disclosures}

The author reports no conflict of interest.

\section{Correspondence}

Aaron A. Cohen-Gadol: acohenmd@gmail.com.

INCLUDE WHEN CITING

Published online December 21, 2018; DOI: 10.3171/2018.9.JNS182475. 